\title{
DETERMINAÇÃO DE SEXO EM ABELHAS. XXX. INFLUÊNCIA DA QUANTIDADE DE ALIMENTO E DO HORMÔNIO JUVENIL NA DETERMINAÇÃO DAS CASTAS EM PARTAMONA CUPIRA HELLERI (HYMENOPTERA, APIDAE, MELIPONINAE)
}

\author{
Lúcio Antônio de Oliveira Campos ${ }^{1}$ \\ Cláudia Diniz Pinto Coelho ${ }^{1}$
}

\begin{abstract}
SEX DETERMINATION IN BEES. XXX. INFLUENCY OF THE QUANTITY OF THE FOOD AND JUVENILE HORMONE IN THE CASTES DETERMINATION IN PARTAMONA CUPIRA HELLERI (HYMENOPTERA, APIDAE, MELIPONINAE). In Partamona cupira, caste determination follows the general patterns of Trigonini bees. The effects of the juvenile hormone and the amount of food on the process were studied. The frequency of queens increased with the quantity of food ingest by the larvae and or whith the amount of juvenile hormone topically applicated. Queens emerged earlier than workers. Intercastes were not found, sugesting that castes differentiation is a canalised process in this species.
\end{abstract}

KEY WORDS. Hymenoptera, Apidae, Meliponinae, Partamona cupira

As abelhas da tribo Trigonini possuem um mecanismo trófico de determinação das castas, de tal modo que as rainhas emergem de células maiores que aquelas das quais emergem as operárias. Nessas células as larvas recebem alimento qualitativamente semelhante aquele dado para as operárias (HERTEFELDER, 1986; CAMARGO, 1972), porém em quantidade maior.

O envolvimento do Hormônio Juvenil (HJ) no processo de diferenciação das castas em abelhas dessa tribo foi mostrado por CAMPOS (1977) por meio de aplicação tópica de $\mathrm{HJ}$ em larvas de operárias de Plebeia nigriceps, Schwarziana quadripunctata, Scaptotrigona sp. e Partamona cupira, na fase de tecelagem do casulo, induzindo a diferenciação destas em indivíduos com características de rainha. O nível de HJ é maior em larvas de rainhas no último instar em Scaptotrigona depilis, que em larvas de operárias (HERTEFELDER, 1986).

$\mathrm{O}$ modo pelo qual a quantidade de alimento ingerido pela larva induz o aumento na síntese de HJ é ainda desconhecido. Entretanto, em Apis melifera, ASSENCOT \& LENSKY (1986) mostraram que a alta concentração de glicose e frutose no alimento larval das rainhas funciona como um fagoestimulante fazendo com que as larvas de rainhas ingiram o alimento com maior rapidez que aquelas de operárias, induzindo uma distenção da parede do intestino, o que

1) Departamento de Biologia Geral, Universidade Federal de Viçosa, 36570-000 Viçosa, Minas Gerais, Brasil. 
por mecanismo reflexo, levaria a um aumento no nível de HJ, como observado por WIGGLESWORTH (1934) em Rhodnius.

CAMPOS (1977) propôs que a ação morfogenética do HJ se dê por ação direta sobre o genoma. Essa ação ocorre em um período sensível da vida larval do inseto, determinando o caráter a se expressar no instar seguinte (NIJOUT e WHEELER, 1982).

O objetivo do presente trabalho foi estudar o processo de determinação de castas em Partamona cupira e a influência do HJ no mesmo.

\section{MATERIAL E MÉTODOS}

As colônias de abelhas utilizadas foram coletadas na região de Viçosa, Minas Gerais e mantidas no apiário da Universidade Federal de Viçosa.

$\mathrm{O}$ alimento utilizado para a criação das larvas foi retirado de células de operárias e/ou machos (as células onde são criados machos e operárias não são distingüíveis em Meliponinae), nas quais os ovos ainda não haviam eclodido. Após a coleta, o alimento foi armazenado sob refrigeração, tendo sido homogenizado no momento da utilização. Esse alimento foi colocado em pequenas cúpulas de cera, com o auxílio de uma pipeta de $0,1 \mathrm{ml}$ acoplada a uma seringa cujo êmbolo era controlado por um parafuso. As quantidades de alimento utilizadas foram dosadas a partir da quantidade existente em células de operárias ( $31 \mu$ l é o valor médio), com o aumento gradativo, isto é, as cúpulas foram aprovisionadas com $35,45,55,65$ e $75 \mu \mathrm{l}$ de alimento sobre o qual foram colocadas larvas recém-eclodidas retiradas de células de operárias com o auxílio de um estilete com ponta dobrada em ângulo de $90^{\circ}$. Essas cúpulas foram fixadas em placas de Petri e depositadas em um cristalizador em cujo fundo foi colocado um pedaço de algodão embebido em água destilada para evitar a desidratação do alimento. $\mathrm{O}$ frasco foi fechado e mantido em estufa incubadora a $28^{\circ} \mathrm{C}$ até que as larvas terminassem de se alimentar.

Ao final da fase de alimentação o $\mathrm{HJ}$ foi aplicado topicamente, ao longo da região lateral da larva com o auxílio de micropipeta de $1 \mu \mathrm{l}$, previamente diluído em acetona de modo a se obter dosagens de 0,5 ou $0,1 \mu \mathrm{g}$ por larva. $\mathrm{O}$ controle foi constituído de larvas tratadas com $1 \mu \mathrm{l}$ de acetona pura. As placas foram então mantidas em estufa a $28^{\circ} \mathrm{C}$, fora do cristalizador.

O diagnóstico das castas foi feito nos adultos com base em características morfológicas que discriminam bem rainhas de operárias.

\section{RESULTADOS E CONCLUSÃO}

Os resultados obtidos (Tab. I) confirmam o trabalho realizado por CAMPOS (1977), no que se refere ao HJ, demonstrando que a determinação de castas em Partamona cupira segue o padrão geral dos Trigonini.

Observa-se que a freqüência de rainhas aumenta à medida que se aumenta a quantidade de alimento ingerido pelas larvas. Nos tratamentos com $55 \mu \mathrm{l}$ de alimento, obteve-se $100 \%$ de operárias. Quando as larvas recebem $65 \mu \mathrm{l}$ 
de alimento a freqüência de rainhas foi de $72,7 \%$. O mecanismo proposto por ASSENCOT \& LENSKY (1976), para Apis mellifera, no que se refere à indução do aumento do título de $\mathrm{HJ}$ estimulado pela distenção do intestino, poderia explicar esse aumento na freqüência de rainhas também nesta espécie.

Tabela I. Freqüência de rainhas e operárias em relação ao número total de fêmeas adultas obtidas a partir de larvas tratadas somente com alimento e alimento mais $\mathrm{HJ}$.

\begin{tabular}{|c|c|c|c|c|c|c|}
\hline \multirow{3}{*}{$\begin{array}{l}\text { Alimento } \\
\qquad(\mu 1)\end{array}$} & \multicolumn{6}{|c|}{ Hormônio Juvenil $(\mu \mathrm{g})$} \\
\hline & \multicolumn{2}{|c|}{$0,0(1 \mu 1$ de acetona $)$} & \multicolumn{2}{|c|}{0,05 (em $1 \mu 1$ de acetona) } & \multicolumn{2}{|c|}{0,1 (em $1 \mu 1$ de acetona) } \\
\hline & Operárias & Rainhas & Operárias & Rainhas & Operárias & Rainhas \\
\hline 35 & $32(100 \%)$ & - & $12(44,4 \%)$ & $15(55,6 \%)$ & $5(20,0 \%)$ & $20(80,0 \%)$ \\
\hline 45 & $19(100 \%)$ & - & $5(14,3 \%)$ & $30(85,7 \%)$ & $2(25,0 \%)$ & $6(75,0 \%)$ \\
\hline 55 & $26(100 \%)$ & - & - & $29(100 \%)$ & - & $24(100 \%)$ \\
\hline 65 & $9(27,35 \%)$ & $24(72,7 \%)$ & - & $27(100 \%)$ & - & $24(100 \%)$ \\
\hline 75 & - & $11(100 \%)$ & - & $12(100 \%)$ & - & $6(100 \%)$ \\
\hline
\end{tabular}

O aumento na freqüência de rainhas ocorre também nos tratamentos com HJ. Nos tratamentos cujas larvas receberam $35 \mu$ l de alimento, a aplicação de $0,05 \mu \mathrm{g}$ de $\mathrm{HJ}$ determinou uma freqüência de $55,6 \%$ de rainhas e a aplicação de $0,1 \mu \mathrm{g}$ aumentou essa freqüência para $80 \%$. CAMPOS (1977) relaciona a obtenção de rainhas, por esse tratamento, com a aceleração da muda de tal forma que esta se dá antes da degeneraçấo dos ovários. Outras características fenotípicas de rainhas também estariam relacionadas com a muda precoce. Neste trabalho as rainhas emergiram de três a quatro dias mais cedo que operárias que receberam o mesmo tratamento.

Em nenhum tratamento foram observadas intercastas, sugerindo que o processo seja canalizado. Neste caso, o nível de HJ no último instar larval, controlado pela quantidade de alimento ingerido pela larva, a conduziu por um dos seguintes caminhos: ou em direção a um fenótipo de rainha (níveis altos de HJ) ou em direção a um fenótipo de operária (níveis baixos de $\mathrm{HJ}$ ), não ocorrendo neste caso indíviduos com características intermediárias como os observados por CAMARGO (1972) em Scaptotrigona postica.

$\mathrm{O}$ fato de larvas alimentadas com quantidades de alimento superiores àquelas recebidas por operárias, porém insuficientes para produzir rainhas (45-55 $\mathrm{l}$ l) e tratadas com HJ em doses que são incapazes de induzir a diferenciação de rainhas a partir de larvas normais de operárias $(0,05 \mu \mathrm{g})$ se diferenciarem em rainha indica que o aumento gradual na quantidade de alimento ingerido leva a um aumento também gradual na concentração de $\mathrm{HJ}$ na larva, de tal modo que em larvas alimentadas com maior quantidade de alimento, uma dose menor de $\mathrm{HJ}$ exógeno é necessária para que seja atingido o limiar de concentração necessário para que a larva se diferencie em rainha. A outra possibilidade, de que a larva que recebesse maior quantidade de alimento apresentasse maior sensibilidade ao $\mathrm{HJ}$, parece ser menos provável, uma vez que, larvas de rainhas possuem maior concentração de $\mathrm{HJ}$ que aquelas de 
operárias (HERTEFELDER, 1986). Uma resposta definitiva poderá ser obtida dosando-se o HJ em larvas alimentadas com diferentes quantidades de alimento.

A mortalidade das larvas foi muito elevada, sendo maior nos tratamentos que receberam $0,1 \mu \mathrm{g}$ de $\mathrm{HJ}$ (Tab. II). Essa elevada mortalidade pode estar relacionada aos efeitos teratogênicos do HJ (CAMPOS, 1977), ou aos efeitos da manipulação.

Tabela II. Mortalidade total das larvas por tratamento com alimento e alimento mais HJ.

\begin{tabular}{cccc}
\hline \multirow{2}{*}{$\begin{array}{c}\text { Alimento } \\
(\mu 1)\end{array}$} & \multicolumn{3}{c}{ Hormônio Juvenil $(\mu \mathrm{g})$} \\
\cline { 2 - 4 } & 0,0 & 0,05 & 0,1 \\
\hline 35 & $28(46,6 \%)$ & $31(51,7 \%)$ & $35(58,0 \%)$ \\
45 & $39(65,0 \%)$ & $23(38,3 \%)$ & $28(70,0 \%)$ \\
55 & $28(46,6 \%)$ & $27(45,0 \%)$ & $30(50,0 \%)$ \\
65 & $20(33,3 \%)$ & $11(27,5 \%)$ & $35(58,3 \%)$ \\
75 & $6(30,0 \%)$ & $8(40,0 \%)$ & $14(70,0 \%)$ \\
\hline
\end{tabular}

AGRADECIMENTOS. Os autores agradecem à Dr. Silvia das Graças Pompolo e ao Dr. W.E. KERR pela leitura do manuscrito e pelas sugestões apresentadas.

\section{REFERÊNCIAS BIBLIOGRÁFICAS}

ASENCOT, M. \& Y. LENSKY. 1976. The effect of sugars and Juvenile Hormone on the differentiation of the female honeybee larvae (Apis mellifera) to queens. Life Sciences 18: 693-700.

CAMARGO, C.A. 1972. Aspectos da Reprodução dos Apídeos Sociais. Tese de Mestrado, não publicada, Universidade de São Paulo, Ribeirão Preto, 63p.

CAMPOS, L.A.DE O. 1977. O Hormônio Juvenil nas Abelhas: Seu Papel na Diferenciação das Castas e nos Aspectos do Controle Social. Tese de Doutorado, não publicada, Universidade de São Paulo, Ribeirão Preto, 67p. HERTEFELDER, K. 1986. Trophogenese Basis und Endokrine Reaktion in der Kastenentwickling bei Stachellosen Bienen. Tese de Doutorado, não publicada, Universidade de Tübingen, Tübingen, 146p.

NIJOUT, H.F. \& D.E. WHEELER. 1982. Juvenile hormone and physiological basis of insect polymorphisms. Quart. Rev. Biol. 57: 109-133.

WIGGLESWORTH, V.B. 1934. The Physiology of Ecdysis in Rhodnius II. Factors controlling moulting and "Methamorphosis". Quart. J. Microscop. Sc. 77: 191-222. 